\title{
Regulation of Photochemical Energy Transfer Accompanied by Structural Changes in Thylakoid Membranes of Heat-Stressed Wheat
}

\section{Yoko Marutani ${ }^{1}$, Yasuo Yamauchi ${ }^{1, *}$, Akihito Miyoshi ${ }^{2}$, Kanako Inoue ${ }^{1}$, Ken-ichi Ikeda ${ }^{1}$, Masaharu Mizutani ${ }^{1}$ and Yukihiro Sugimoto ${ }^{1}$}

1 Graduate School of Agricultural Science, Kobe University, 657-8501 Kobe, Japan;

E-Mails: yoko.marutani@stu.kobe-u.ac.jp (Y.M.); i-kana@people.kobe-u.ac.jp (K.In.);

ikeken@phoenix.kobe-u.ac.jp (K.Ik.); mizutani@gold.kobe-u.ac.jp (M.M.); yukihiro@kobe-u.ac.jp (Y.S.)

2 Faculty of Agriculture, Kobe University, 657-8501 Kobe, Japan;

E-Mail: miyoshi.akihito.53v@st.kyoto-u.ac.jp

* Author to whom correspondence should be addressed; E-Mail: yamauchi@kobe-u.ac.jp; Tel./Fax: +81-78-803-5886.

External Editor: Marcello Iriti

Received: 30 September 2014; in revised form: 4 December 2014 / Accepted: 5 December 2014 / Published: 11 December 2014

\begin{abstract}
Photosystems of higher plants alleviate heat-induced damage in the presence of light under moderate stressed conditions; however, in the absence of light (i.e., in the dark), the same plants are damaged more easily. (Yamauchi and Kimura, 2011) We demonstrate that regulating photochemical energy transfer in heat-treated wheat at $40{ }^{\circ} \mathrm{C}$ with light contributed to heat tolerance of the photosystem. Chlorophyll fluorescence analysis using heat-stressed wheat seedlings in light showed increased non-photochemical quenching (NPQ) of chlorophyll fluorescence, which was due to thermal dissipation that was increased by state 1 to state 2 transition. Transmission electron microscopy revealed structural changes in thylakoid membranes, including unstacking of grana regions under heat stress in light. It was accompanied by the phosphorylation of thylakoid proteins such as D1 and D2 proteins and the light harvesting complex II proteins Lhcb1 and Lhcb2. These results suggest that heat stress at $40{ }^{\circ} \mathrm{C}$ in light induces state 1 to state 2 transition for the preferential excitation of photosystem I (PSI) by phosphorylating thylakoid proteins more strongly. Structural changes of thylakoid membrane also assist the remodeling of photosystems and regulation
\end{abstract}


of energy distribution by transition toward state 2 probably contributes to plastoquione oxidation; thus, light-driven electrons flowing through PSI play a protective role against PSII damage under heat stress.

Keywords: heat stress; state transition; wheat; thylakoid membrane; photosystem; phosphorylation

\section{Introduction}

High temperatures under recent climate changes is a major environmental constraint for plant production because photosynthesis, which includes photochemical reactions as well as carbon assimilation, is a very heat-sensitive process [1]. Rubisco activase is an enzyme that maintains Rubisco in an active state during the carbon assimilation process and is highly sensitive to heat denaturation. Inactivation of Rubisco activase leads to a limitation during carbon assimilation [2]. Photosystem II (PSII) photochemical reactions exhibit higher susceptibility to heat stress [3]. Heat stress affects electron flow both on the donor side and the acceptor side of PSII. The water-oxidation system of PSII is believed to be the first step to be damaged by heat stress [4]. The electron flow from $Q_{A}$ to $Q_{в}$ in PSII has also been reported to be damaged at high temperatures [5]. In contrast, photosystem I (PSI) is more heat tolerant than PSII [6].

Plants have multiple protective mechanisms against heat stress, including the induction of heat shock proteins to protect PSII from heat-induced damage by stabilizing protein folding and preventing protein aggregation $[7,8]$, isoprene emissions to stabilize thylakoid membranes [9-11], and a reactive oxygen species scavenging system mediated by antioxidants such as ascorbic acid, tocopherols, and glutathione [12]. In addition, a change in energy transfer in photosystems is believed to alleviate heat-induced damage, e.g., thermal dissipation and state transition [10].

State transition is a well-studied phenomenon that occurs in changing light conditions in photosynthetic organisms such as higher plants, cyanobacteria and green algae. However, the light condition that induces state transition differs among species. In green algae, which have been extensively studied, it is believed to be an acclimation response that redistributes excitation energy between PSII and PSI in the short term [13]. In contrast, it has been recently demonstrated that state transition is a long-term acclimation to various natural light conditions in higher plants and that a part of the light-harvesting chlorophyll-binding protein II (LHCII) is phosphorylated and behaves as an effective PSI antenna [14]. State transition is triggered by changes in the redox state of the plastoquinone (PQ) pool. Reduction of PQ activates LHCII phosphorylation, which initiates the subsequent migration of LHCII to PSI (state 1 to state 2 transition). Thus, various environmental stressors in higher plants, including heat stress, tend to affect state transition through a change in energy distribution between the photosystems [15]. Moderate heat stress induces increased energy transfer to PSI at the expense of PSII [16] and migration of phosphorylated LHCII from grana stack to stroma lamellae [17], suggesting a state 1 to state 2 transition, with LHCIIs migrating from PSII to PSI.

In this study, we focused on the regulation of photochemical energy transfer with structural and biochemical changes in thylakoid membranes in response to heat stress using normal-growing wheat, 
heat-stressed wheat in the presence of light, and wheat recovering from heat stress. The results revealed that heat stress induces state 2; we observed increased non-photochemical quenching (NPQ) of chlorophyll fluorescence, LHCII phosphorylation and unstacked grana regions in thylakoid membranes in response to heat stress.

\section{Results and Discussion}

\subsection{Increase in NPQ (Non-Photochemical Quenching) of Chlorophyll Fluorescence and Unstacked} Region in Thylakoid Membranes under Heat Stress

A temperature of approximately $40{ }^{\circ} \mathrm{C}$ is perilous for many plant species [18]. Also in wheat plants, $40{ }^{\circ} \mathrm{C}$ is a potentially critical temperature for decreasing photosynthetic activity. We previously reported the mechanism for the damage caused by a rapid decrease in the maximum photochemical quantum yield of PSII $\left(F_{v} / F_{m}\right)$ in seedlings within $1 \mathrm{~h}$ of heat treatment at $40{ }^{\circ} \mathrm{C}$ in the absence of light [19]. Our data suggest that in the dark, the enhanced introduction of reducing power from stroma into thylakoid membranes that occurs at temperatures $>40{ }^{\circ} \mathrm{C}$ causes over-reduction of $\mathrm{PQ}$, resulting in damage to the $\mathrm{D} 1$ protein. However, $F_{v} / F_{m}$ did not decrease under a $40{ }^{\circ} \mathrm{C}$ heat treatment in light [19], indicating that light drives a system that protects PSII from damage caused by over-reduction of PQ at high temperatures.

To assess the change in energy distribution of photochemical reactions in heat-stressed wheat seedlings in the presence of light, we performed chlorophyll fluorescence analysis under different temperatures (Figure 1a,b). NPQ increased after $30 \mathrm{~min}$ of a $40{ }^{\circ} \mathrm{C}$ heat treatment (L40) compared with that under the normal condition (L25). The higher NPQ in L40 decreased to the same level as that of L25 after 30-min recovery at $25{ }^{\circ} \mathrm{C}$ in light (R25), whereas $F_{v} / F_{m}$ was not affected by heat treatment in the light (Figure 1a). NPQ is subdivided into three components related to the thermal dissipation of excessive light energy (qE), state transition (qT), and photoinhibition (qI). $\mathrm{qE}$ and $\mathrm{qT}$ were major components for increasing the L40 NPQ. Thermal dissipation mediated by the xanthophyll cycle is facilitated under heat conditions to protect PSII from excess light energy [20]. An activated cyclic electron flow around PSI enhances thermal dissipation under heat stress and supports the acidification of the lumen to accelerate the xanthophyll cycle [21]. Although qT was the largest in L40, it was detected even under normal conditions in L25 and R25, coinciding with a recent report that part of the mobile LHCII in Arabidopsis is associated with PSI under all natural light conditions for plants [14]. The increasing qT in L40 suggests that heat treatment in light induces state 2 when more LHCIIs are associated with PSI. 
Figure 1. Heat treatment in light induced increase of NPQ (non-photochemical quenching) and unstacking of thylakoid membrane. (a) PSII (Photosystem II) maximum quantum efficiency $\left(F_{v} / F_{m}\right)$. One-week-old wheat seedlings were heat-stressed under light (100 $\mu \mathrm{mol}$ photons $\mathrm{m}^{-2} \cdot \mathrm{s}^{-1}$; L40). L40 wheat recovered at $25{ }^{\circ} \mathrm{C}$ was used as R25. Non-heat-stressed wheat was used as the control L25 plant. Actinic light (AL), $190 \mu \mathrm{mol}$ photons $\mathrm{m}^{-2} \cdot \mathrm{s}^{-1}$. Error bars indicate \pm standard errors (SEs) $(n=3)$; (b) Non-photochemical quenching (NPQ), qE, qT, and qI of each sample. AL, $820 \mu \mathrm{mol}$ photons $\mathrm{m}^{-2} \cdot \mathrm{s}^{-1}$; (c) Electron micrographs of wheat leaves: left panel, control plant; middle panel, plant heat-stressed in light $\left(100 \mu \mathrm{mol}\right.$ photons $\left.\mathrm{m}^{-2} \cdot \mathrm{s}^{-1}\right)$; right panel, plant recovered in light from heat stress at $25{ }^{\circ} \mathrm{C}$. Single and double asterisks indicate a starch granule and a plastoglobule, respectively. SL, stroma lamellae. G, Grana. Bars $=500 \mathrm{~nm}$; and (d) Graphs: top panel, percentage of stacked and unstacked isolated wheat thylakoid membranes. $(n=3)$; Upper middle panel, height of 10 thylakoid layers (nm); Lower middle panel, starch granules in a chloroplast; bottom panel, numbers of plastoglobules in each chloroplast. Error bars indicate \pm SEs $(n=50)$. Note that L40 chloroplasts altered thylakoid membranes but not those of L25 and R25.

(a)

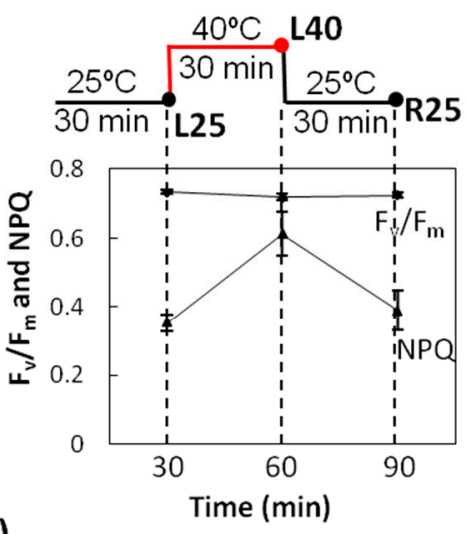

(c)

$\mathrm{L} 25^{\circ} \mathrm{C}$

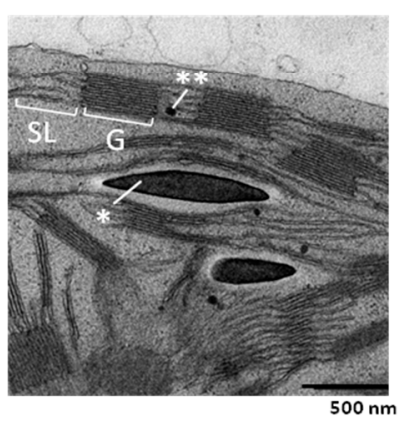

(b)

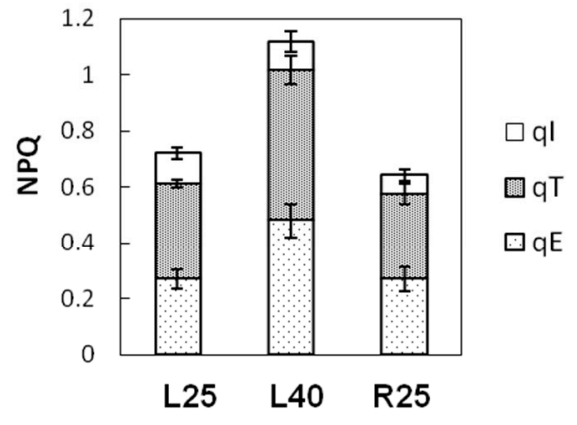

$\mathrm{L} 40^{\circ} \mathrm{C}$

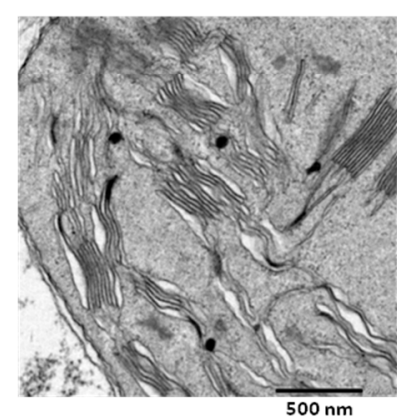

$\mathrm{R} 25^{\circ} \mathrm{C}$

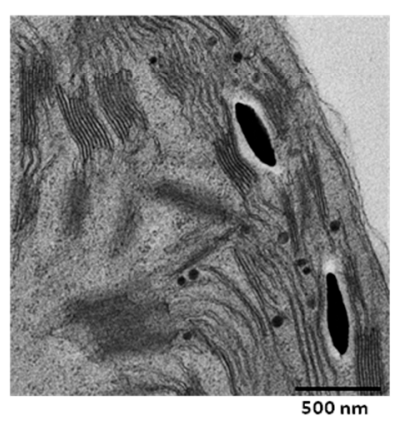

(d)
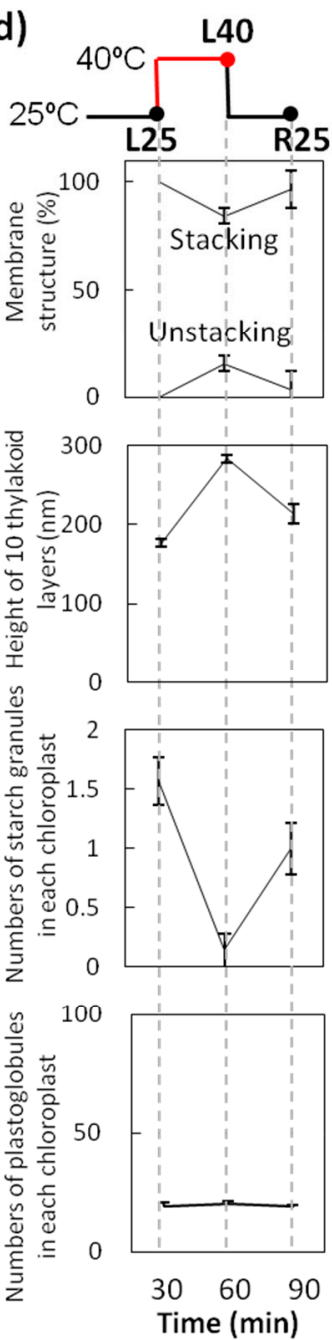
Previous studies indicate that state transition and thylakoid membrane structure are closely related [22,23]. Transmission electron microscopy (TEM) was used to investigate the changes in the thylakoid membrane ultrastructure among various state 2 conditions in leaf chloroplasts. Thylakoid membranes in higher plants are mainly composed of two regions called appressed grana stacks and non-appressed stroma lamellae. Thylakoid membranes in L25 seedlings formed well-distinguished appressed grana stacks and stroma lamella (Figure 1c). However, many grana clearly unstacked after L40 treatment. These dissociated grana stacks seemed to be restacked within 30 min under normal conditions after heat stress, as shown in the R25 sample (Figure 1c,d). However, the height of the 10 thylakoid layers clearly differed among the three samples, i.e., the L40 grana region was mostly unstacked, while the R25 grana region was more loosely stacked than the L25 grana region (Figure 1d). These results suggest that L40 is a condition in which more LHCII is associated with PSI among these three samples. Starch granules were observed in L25; however, very few starch granules were observed after heat treatment (Figure 1c,d). This result may be related to the increased activity of starch degradation. The activity of $\beta$-amylase, which is involved in starch degradation, increased under moderate heat conditions [24]. It is likely that L40 stimulates the activity of several enzymes involved in starch degradation such as $\beta$-amylase. In addition, the decrease of starch granules in L40 would be related to the inability to make starch because of an inactivated Calvin-Benson cycle at high temperatures. The production of starch granules recovered in R25 after heat stress. Plastoglobules, which are plastid-localized lipoprotein particles containing tocopherols and other lipid isoprenoid-derived metabolites, increase in number under moderate heat conditions in A. thaliana [25]. However, the numbers of plastoglobules in chloroplasts of wheat did not differ among the three samples (Figure 1d).

\subsection{Phosphorylation Level of Thylakoid Proteins Increased by Heat Treatment}

The increase in NPQ and qT observed in chlorophyll fluorescence analysis (Figure 1a,b) suggested that state 2 occurred in the L40 samples. During state 2 induction, PSII core proteins and LHCII are phosphorylated by thylakoid kinase STN7 and STN8, respectively [13]. To compare the phosphorylation levels of thylakoid proteins in heat-treated wheat, we performed immunobloting using anti-phosphothreonine (pThr) antibody because Thr residues are key phosphorylated amino acid residues [26]. Anti-pThr signals increased at approximately 33 and $25 \mathrm{kDa}$ in the L40 samples compared with those in the L25 samples (Figure 2a). These phosphorylated thylakoid proteins were purified using the ProQ phosphorylated protein purification kit and separated by sodium dodecyl sulfate polyacrylamide gel electrophoresis (SDS-PAGE). Several bands appearing at approximately 23-35 kDa were fractionated into two fractions (Fr. A and B) and identified by peptide mass spectrometry (MS) fingerprinting analysis (Figure 2b). The sequence in fraction A was identified as D1 and D2 proteins. The sequences in Fr. B were identified as three LHCIIs, as CP26, Lhcb1, and Lhcb2 (Table S1). 
Figure 2. Phosphorylation level of PSII proteins increased by heat treatment. (a) Thylakoid membrane proteins were subjected to SDS-PAGE and subsequent immunoblotting using specific phospho-threonine antibodies; (b) Identification of the phosphorylated thylakoid proteins. Purified phosphorylated thylakoid proteins in L40 that were purified using the ProQ Diamond Phosphoprotein Enrichment Kit were separated by SDS-PAGE, and the bands in the dotted boxes were detected by peptide MS finger printing analysis. Mascot search results are shown in Supplemental Table S1; and (c) Thylakoid membrane proteins, stacked grana and unstacked stroma lamellae were subjected to SDS-PAGE and subsequent immunoblotting using specific phospho-threonine antibodies.

(a)

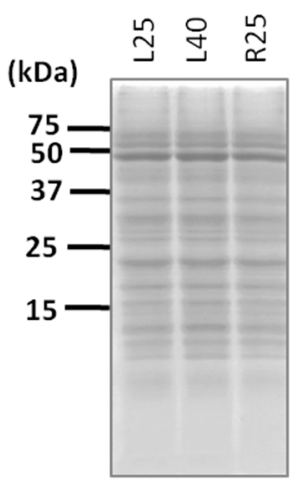

CBB
$\stackrel{\Xi}{g} \stackrel{9}{\dddot{2}}$

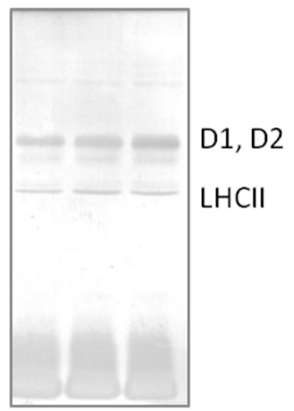

Anti-pThr

(b)

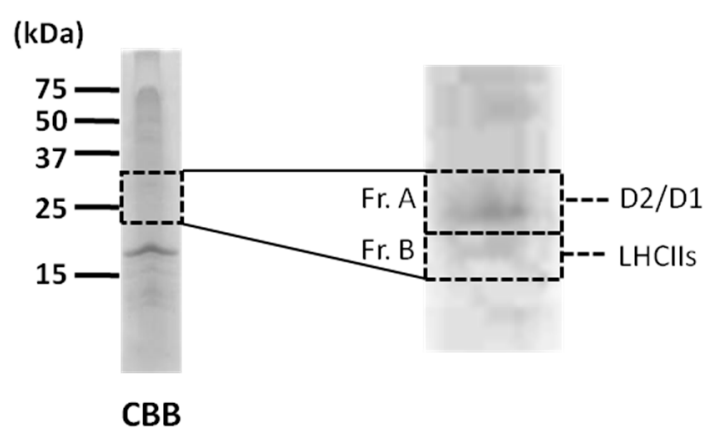

(c)
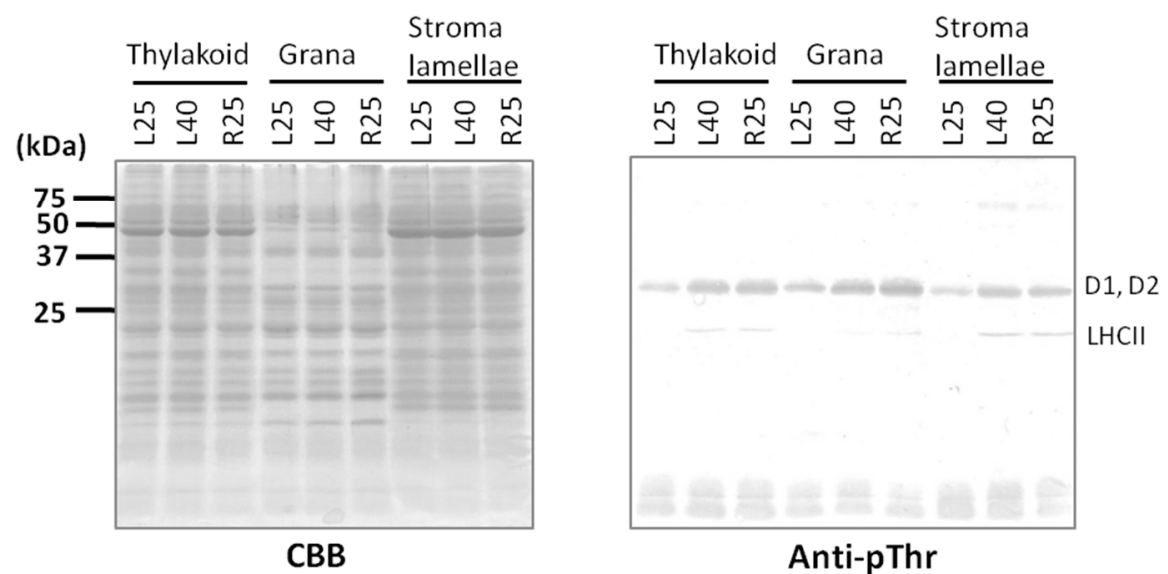

The phosphorylation of LHCII is important for it to dissociate from PSII, which is enriched in the grana region, and is associated with PSI in stroma lamellae during state transition. To identify the location of the phosphorylated PSII core and LHCII proteins detected in the heat-treated samples, we separated thylakoid membranes into grana and stroma lamellae by digitonin fractionation and analyzed the fractions by SDS-PAGE. Heat treatment did not affect the protein profiles of any of the samples (Figure 2c, Coomassie Brilliant Blue (CBB) staining). Many phosphorylated LHCIIs were detected in both L40-treated and R25-treated stroma lamellae (Figure 2c, immunoblot). D1 and D2 proteins were detected in a much more phosphorylated state in the L40 and R25 grana. The phosphorylation of D1 and D2 proteins is believed to be correlated with the thylakoid membrane structure because the negative charge on the phosphate groups influences the surface charges on the thylakoid membranes, resulting in unstacked, appressed membrane regions caused by electrostatic repulsion $[23,27]$. Therefore, state transitions and the thylakoid membrane ultrastructure have been 
suggested to be closely related [22], and an increase in the unstacked grana region has been observed in light-induced state- 2 chloroplasts. Also in heat-induced state 2 in wheat, it is possible that a relationship exists between the phosphorylation of thylakoid proteins and unstacking of thylakoid membranes.

\subsection{Dephosphorylation of Thylakoid Proteins Was Retarded during Recovery from Heat Stress}

The phosphorylation of thylakoid proteins (particularly PSII core proteins such as D1 and D2 proteins) in Arabidopsis under state 2 conditions plays an important role in forming unstacked thylakoid membranes [27]. However, we unexpectedly detected retarded dephosphorylation of these proteins during the 30-min recovery after heat stress (Figure 2c). It is possible that the dephosphorylation of thylakoid proteins is not important for the reconstitution of the stacked membranes. We measured the protein phosphorylation level during a prolonged recovery for $24 \mathrm{~h}$ to confirm the time required to dephosphorylate the PSII core and LHCII proteins (Figure 3). Phosphorylated D1/D2 and LHCII proteins were detected using anti-pThr antibody, and phosphorylated D1/D2 proteins were quantified by densitometric analysis. As shown in Figure 3a, compared with samples under normal conditions (L25), the relative phosphorylation levels of D1/D2 proteins increased under the $40{ }^{\circ} \mathrm{C}$ treatment in light (L40). The amount of D1/D2 phosphorylated proteins was the highest at $25^{\circ} \mathrm{C}$ during $0.5-1 \mathrm{~h}$ after heat stress. The 4-8 h heat stress-recovered samples showed comparable phosphorylation levels with L25. A similar result was observed in Lhcb2 dephosphorylation analysis. Phosphorylated thylakoid proteins were separated by Phos-tag TM SDS-PAGE. In this system, phosphorylated proteins interact with Phos-tag ${ }^{\text {TM }}$ co-polymerized with acrylamide in the gel, resulting in a slower migration of phosphorylated proteins than non-phosphorylated proteins [28]; thus, the ratio of phosphorylated proteins could be easily estimated. It has been reported that Lhcb2 phosphorylation plays a crucial role in inducing state 2 [29,30]; thus, we used Lhcb2 antibody to determine the Lhcb2 phosphorylation ratio in heat-stressed wheat. Densitometric analysis suggested that the shifted band occupied 19.2\% of Lhcb2 before the L40 treatment, which increased to $26.4 \%$ of Lhcb2 (Figure 3b). More than $8 \mathrm{~h}$ were required to decrease phosphorylation to the normal level during recovery at $25{ }^{\circ} \mathrm{C}$ in light. These results suggest that the dephosphorylation of thylakoid proteins is not correlated with the reconstitution of stacked membranes during recovery at $25{ }^{\circ} \mathrm{C}$ from heat stress. This retarded dephosphorylation of thylakoid proteins could be responsible for the phosphatase of thylakoid proteins such as PPH1/TAP38 for LHCII [31,32] and PBCP for PSII core proteins [33]. The dephosphorylation process of thylakoid proteins is believed to be independent of the redox state of PQ. Therefore, reduction of the PQ pool seemed to be long-continued during recovery at $25^{\circ} \mathrm{C}$ from heat stress, because the balance between the activities of these kinases and phosphatases determines the phosphorylation state of thylakoid proteins. 
Figure 3. D1 and Lhcb2 were dephosphorylated during the $8 \mathrm{~h}$ recovery. (a) Proteins in the thylakoid membranes were subjected to SDS-PAGE and subsequent immunoblotting using specific phospho-threonine antibodies. Relative phosphorylation levels of D1 proteins are shown at right. $x$-axis is for the time after recovery from heat stress at $40{ }^{\circ} \mathrm{C}$ in light; (b) Thylakoid proteins were separated using Phos-tagTM SDS-PAGE and analyzed by immunoblotting with anti-Lhcb2 antibody. Filled arrowhead and open arrowhead shows the phosphorylated and non-phosphorylated proteins, respectively. Right panel represents the ratio of phosphorylated and non-phosphorylated Lhcb2. $x$-axis is for the time after recovery from heat stress at $40{ }^{\circ} \mathrm{C}$ in light.

(a)

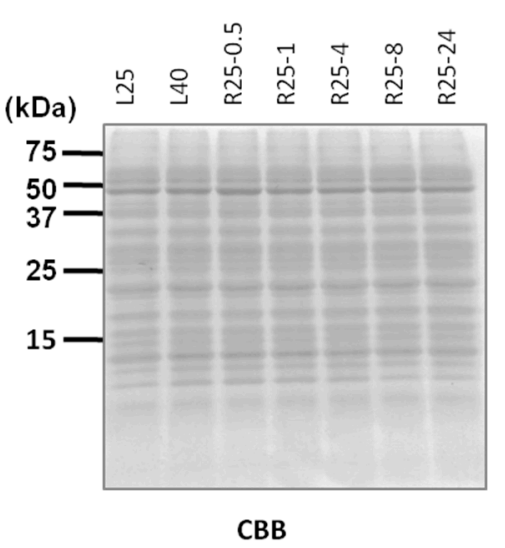

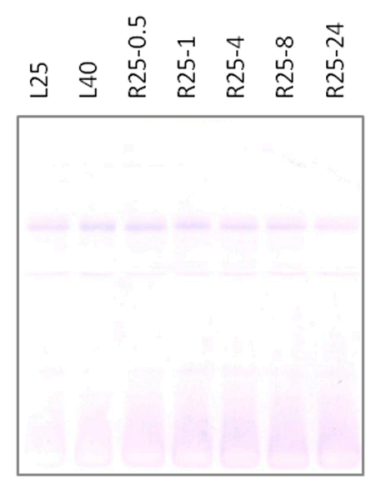

Anti-pThr

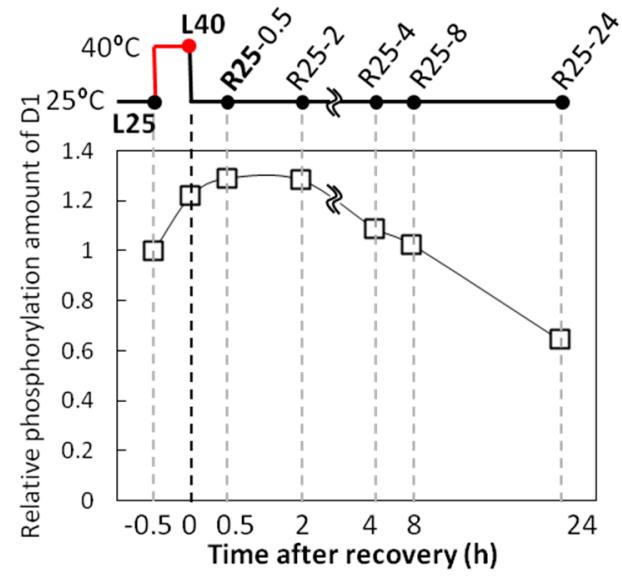

(b)
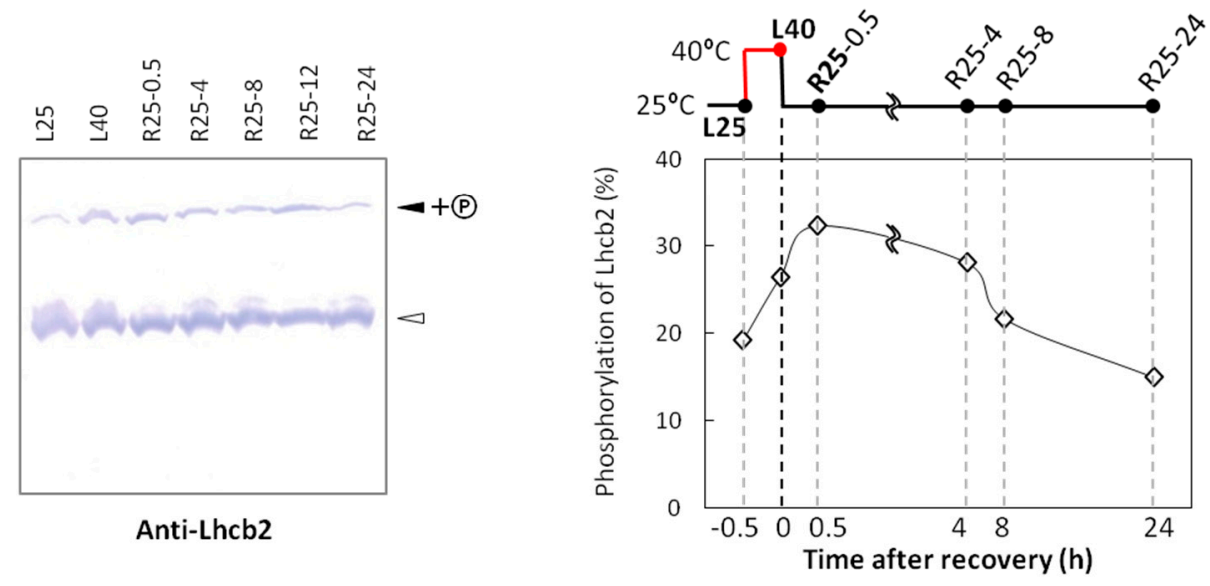

\subsection{PSI-LHCII Supercomplex Increased Following Heat Stress in Light}

Chlorophyll fluorescence and phosphoprotein analyses of wheat leaves showed that heat stress under moderate light induced state 2 , which was much higher than that under growth condition in light, such as L25. In state 2, LHCII, which is bound to PSII in state 1, migrates from PSII and associates with PSI to form the PSI-LHCII supercomplex [34]. To obtain clearer evidence that L40 is in state 2, we compared the amount of the PSI-LHCII supercomplex in L40 to that in state 1 or state 2 induced by a conventional way to provoke state transition. As the conventional way to induce state transition, we used the conditions either after overnight dark adaptation at $25{ }^{\circ} \mathrm{C}$ (state 1) or after $50 \mathrm{~min}$ of $20 \mu \mathrm{mol}$ photons $\mathrm{m}^{-2} \cdot \mathrm{s}^{-1}$ white light treatment at $25{ }^{\circ} \mathrm{C}$ (state 2) [35]. As shown in Figure 4, thylakoid membranes were solubilized using a very mild solubilization condition ( $1 \%$ digitonin and $0.1 \% n$-dodecyl- $\alpha$-D-maltoside $(\alpha-D M))$, which efficiently detects PSI-LHCII supercomplexes [35]. As a result, the amount of the 
PSI-LHCII supercomplex in the L40 sample was similar to that in conventionally induced-state 2 (St2). We also detected an increase in PSI-LHCII phosphorylation using p-Thr antibody, indicating that phosphorylated LHCII is bound to PSI in L40. In addition, we unexpectedly detected that the amount of the PSI-LHCII supercomplex in L25 was closer to that in St2 or L40 rather than that in conventionally induced state $1(\mathrm{St} 1)$.

Figure 4. PSI-LHCII supercomplex increased by heat stress in light. Thylakoid membrane proteins were solubilized with $0.1 \% n$-dodecyl- $\alpha$-D-maltoside $(\alpha-\mathrm{DM})$ and $1 \%$ digitonin and subjected to Blue Native-Polyacrylamide Gel Electrophoresis (BN-PAGE) and subsequent immunoblotting using specific phospho-threonine antibodies.

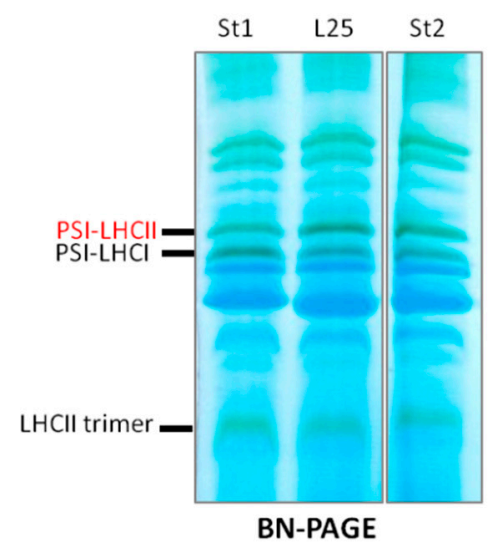

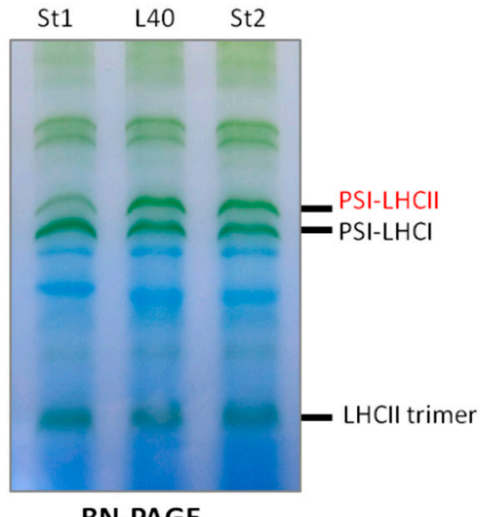

BN-PAGE

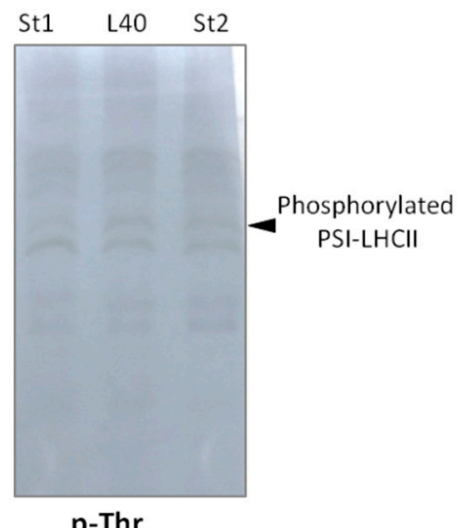

p-Thr

Figure 5 shows a hypothetical scheme of remodeling of photosystems in response to heat. Under growth conditions in light, qT and phosphorylation of thylakoid proteins were detected, and the amount of the PSI-LHCII supercomplex was similar to that in conventionally induced state 2, although linear electron flow (LEF) generates NADPH (Figure 5; upper panel). In this state, part of LHCII is phosphorylated and is associated with PSI in stroma lamellae to keep the PQ pool oxidized by smooth electron flow through PSI, and light energy would preferentially excite PSII [19]. Under high temperature conditions in light, energy distribution between the photosystems and electron transfer would presumably change. At the onset of $40^{\circ} \mathrm{C}$, the enhanced introduction of stromal reducing power into PQ may trigger additional phosphorylation of thylakoid proteins by activated protein kinase such as STN7 and STN8. The increased thylakoid protein phosphorylation level induces thermal dissipation increased by state transition, which was shown to coincide with the unstacking of thylakoid membranes (Figure 5; middle panel). In a previous study, we explained the over-reduction of the PQ pool at $40{ }^{\circ} \mathrm{C}$ in dark [19], which would breakdown oxygen-evolving complex via reactive oxygen species. The Mn cluster is a fragile complex system that can be easily destroyed by heat. Therefore, light would protect the Mn cluster by pumping protons into the lumen to induce thermal dissipation of light energy via the activation of the xanthophylls cycle. During recovery from heat stress, the phosphorylation of thylakoid proteins is maintained, and moderate unstacking of thylakoid membranes may be controlled by the balance between the ST7 and STN8 kinases and the PPH1/TAP38 and PBCP phosphatases. In this state, the energy distribution between the photosystems seems to be the same as that under normal conditions; however, it takes approximately eight hours to return to the original level of thylakoid phosphorylation under normal conditions. 
Figure 5. Schematic diagram of the thylakoid membrane and electron flow under different temperature conditions. Upper panel, at $25{ }^{\circ} \mathrm{C}$ in the light, the linear electron flow (gray arrow) is the primary pathway although most of LHCII is associated with PSI, and the thylakoid membrane is stacked; Middle panel, under heat-stressed condition at $40{ }^{\circ} \mathrm{C}$ in light, LHCIIs and PSII core subunits are phosphorylated. PSI, which is associated with phosphorylated LHCII trimers is preferentially excited and enhances cyclic electron flow (CEF) (gray bold arrow) to avoid over-reduction of PQ. Acidification of lumen by CEF (black arrow) induces PSII thermal dissipation (orange arrow), which restricts linear electron flow (gray thin arrow); Lower panel, recovery from heat stress, LHCIIs and PSII core subunits remain phosphorylated, and the thylakoid membranes are not rigidly appressed.

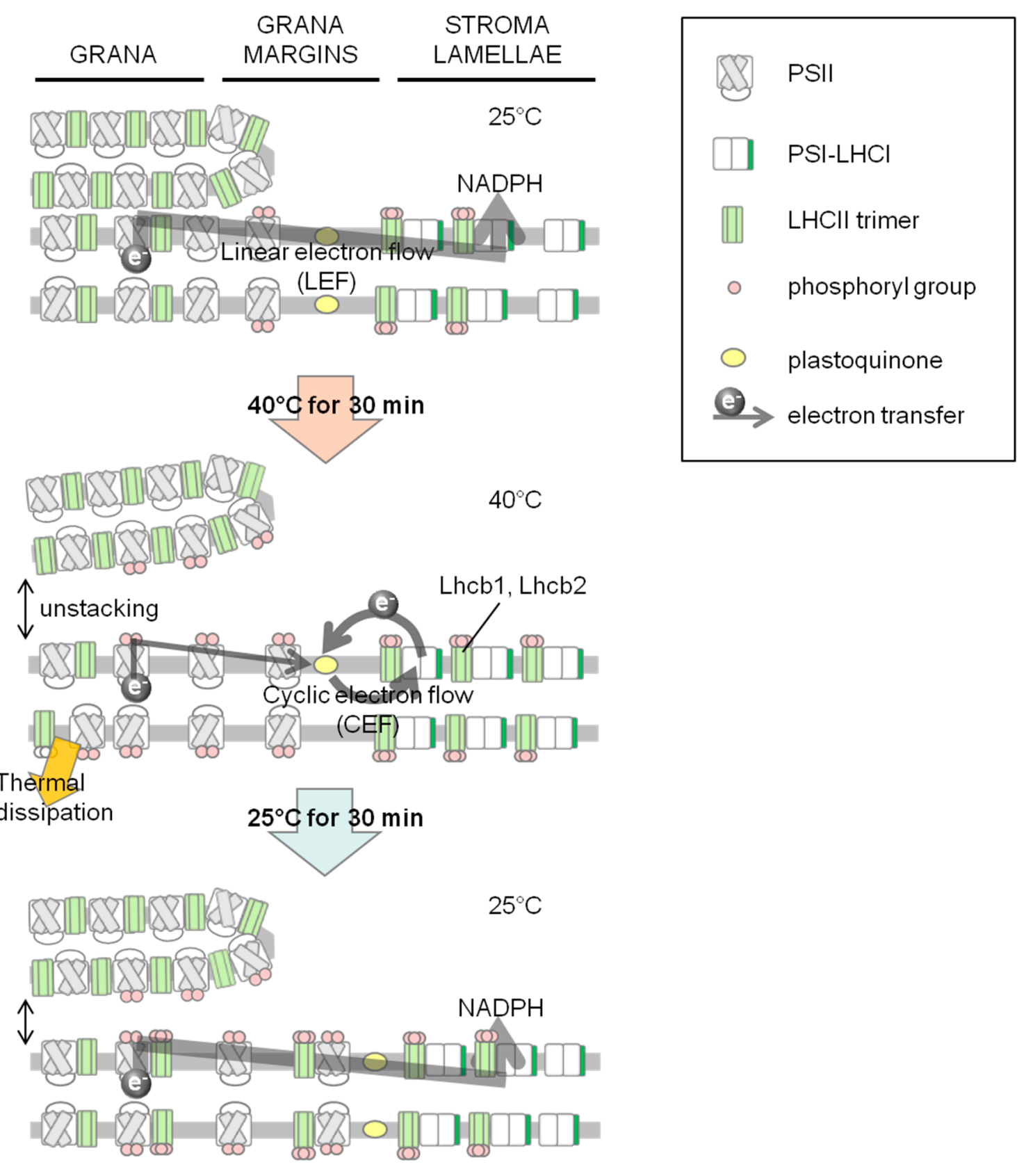




\section{Experimental Section}

\subsection{Plant Materials and Heat Treatments}

Wheat (Triticum aestivum) seedlings were grown on Jiffy-7 (Sakata Seed, Yokohama, Japan) in a growth chamber $\left(12 \mathrm{~h}\right.$ of white light $\left(100 \mu \mathrm{mol}\right.$ photons $\left.\mathrm{m}^{-2} \cdot \mathrm{s}^{-1}\right), 25^{\circ} \mathrm{C}$, Biotron LH-220S, Nippon Medical \& Chemical Instruments, Tokyo, Japan). Heat treatment was carried out by incubating wheat leaves at $40{ }^{\circ} \mathrm{C}$ under fluorescent white light illumination $\left(100 \mu \mathrm{mol}\right.$ photons $\left.\mathrm{m}^{-2} \cdot \mathrm{s}^{-1}\right)$. After heat treatment, plants were recovered for $30 \mathrm{~min}$ at $25{ }^{\circ} \mathrm{C}$ in light. Chemicals used in this research were generally of reagent grade from Wako Pure Chemical Industries (Osaka, Japan).

\subsection{Induction of Conventional State Transition}

As the conventional way to provoke state transition, we used the conditions previously described in [35]. State 1 was induced by overnight dark adaptation at $25^{\circ} \mathrm{C}$. State 2 was induced by 50 min of $20 \mu \mathrm{mol}$ photons $\mathrm{m}^{-2} \cdot \mathrm{s}^{-1}$ white light treatment at $25^{\circ} \mathrm{C}$.

\subsection{Measurement of the Maximal Photochemical Quantum Yield of PSII and Chlorophyll}

Fluorescence Analysis

The maximal photochemical quantum yield of PSII was evaluated from fluorescence measurements. The ratio of variable $\left(F_{v}=F_{m}-F_{o}\right)$ to maximum fluorescence $\left(F_{m}\right)$ was measured using a pulse-modulated fluorometer (Junior-PAM, Heinz Walz GmbH, Effeltrich, Germany); it reflects the quantum yield of PSII [5]. Sample leaves were dark-adapted for $2 \mathrm{~min}$ before the measurements. Dependence of PSII quantum efficiency (ФII), electron transport rate (ETR), and NPQ of the chlorophyll fluorescence on light intensity was calculated as $\left(F_{m}{ }^{\prime}-F_{s}\right) / F_{m}{ }^{\prime}$, $\Phi$ II $\times$ PFD (photon flux density) $\times 0.84 \times 0.5$, and $\left(F_{m}-F_{m}\right) / F_{m}^{\prime}$, respectively [36]. Note that the fractional absorption is assumed here to be 0.84 , and the ratio of PSII to PSI is assumed to be $1: 1$; thus, care must be exercised in making conclusions due to these two assumptions. Blue LED (wavelength of maximum emission: $450 \mathrm{~nm}$ ) was used as the light source. A sample leaf detached from a dark-adapted plant was placed on a wet paper towel, and the temperature was controlled by a heat block. After waiting for $5 \mathrm{~min}$ to equilibrate temperature, chlorophyll fluorescence analysis was performed using the WinControl Program (version 3.1, Walz). Event sequence and light conditions were as follows: measuring light on $\left(0 \mathrm{~min}, 10 \mu \mathrm{mol}\right.$ photons $\left.\mathrm{m}^{-2} \cdot \mathrm{s}^{-1}\right)$, saturating pulse $\left(0.5 \mathrm{~min}, 10,000 \mu \mathrm{mol}\right.$ photons $\left.\mathrm{m}^{-2} \cdot \mathrm{s}^{-1}\right)$, actinic light on (1.5 min, 190), saturating pulse (5 min, 10,000 $\mu \mathrm{mol}$ photons $\mathrm{m}^{-2} \cdot \mathrm{s}^{-1}$ ), actinic light off (5.5 min), and measuring light off. For the detection of $\mathrm{qE}, \mathrm{qT}$, and $\mathrm{qI}$, wheat leaves were illuminated with $820 \mu \mathrm{mol}$ photons $\mathrm{m}^{-2} \cdot \mathrm{s}^{-1}$ of actinic light. NPQ can be detected as the difference between $F_{m}$ and the measured maximal fluorescence after a saturating light pulse during illumination of actinic light $\left(F_{m}{ }^{\prime}\right)$. NPQ is composed by "fast", "middle" and "slow" quench relaxes [37]. After switching off the light, the recovery of $F_{m}$ ' within 2 min and 40 min was regarded as the relaxation of the qE and qT components of NPQ, respectively in wheat. qI component detected as the difference between $F_{m}$ and the recovery of $F_{m}{ }^{\prime}$ within $40 \mathrm{~min}$. qE, qT and $\mathrm{qI}$ values were expressed the percentages of total $\mathrm{NPQ}, \mathrm{NPQ}=\mathrm{qE}+\mathrm{qT}+\mathrm{qI}$. qE, qT and qI were calculated as shown in Müller, et al. [38]. 


\subsection{Preparation of Thylakoid Membranes}

Wheat leaves were homogenized with the following medium: $20 \mathrm{mM}$ Tricine- $\mathrm{NaOH}, \mathrm{pH}$ 7.9, $0.4 \mathrm{M}$ sucrose, and $10 \mathrm{mM} \mathrm{NaCl}$. The homogenate was filtered with two layers of gauze, and then the filtrate was centrifuged at $1000 \times \mathrm{g}$ for $1 \mathrm{~min}$. After the precipitate was discarded, the supernatant was centrifuged at $1500 \times \mathrm{g}$ for $7 \mathrm{~min}$ and the resulting precipitate was used as thylakoid membranes. All procedures were performed below $4{ }^{\circ} \mathrm{C}$ in a dark room under a green light.

\subsection{Digitonin Fractionation of Thylakoid Membranes}

We used digitonin to separate stacked and unstacked thylakoids. Digitonin fractionation of thylakoid membranes and estimation of the degree of thylakoid stacking were performed as reported by Khatoon et al. [39]. Thylakoid membranes were suspended in $250 \mu \mathrm{g} \mathrm{Chl} / \mathrm{mL}$ in a solution containing $0.2 \mathrm{M}$ sorbitol, $1.5 \mathrm{mM} \mathrm{K}_{2} \mathrm{HPO}_{4}\left(\mathrm{pH} \mathrm{7.0)}\right.$, and $10 \mathrm{mM} \mathrm{NaF}$. After incubation at $4{ }^{\circ} \mathrm{C}$ for $15 \mathrm{~min}$, the thylakoid membranes were treated with $0.5 \%$ digitonin for $30 \mathrm{~min}$ on ice. Thylakoid membranes were six-fold diluted with the same solution and centrifuged at $10,000 \times \mathrm{g}$ for $30 \mathrm{~min}$. The amount of chlorophyll in the pellet containing stacked grana relative to that in total thylakoids represented the degree of thylakoid stacking. The supernatant was used as unstacked stroma lamellae that most PSI was recovered.

\subsection{Protein Analysis}

Protein was applied to SDS-PAGE using 12\% (w/v) acrylamide gel. Phos-tag ${ }^{\text {TM }}$ SDS-PAGE was performed using $8 \%(w / v)$ acrylamide gel containing $20 \mu \mathrm{M}$ Phos-tag ${ }^{\mathrm{TM}}$ AAL-107 (Wako Pure Chemical Industries, Osaka, Japan) and $40 \mu \mathrm{M} \mathrm{MnCl}$. After electrophoresis, Phos-tag ${ }^{\text {TM }}$ SDS-PAGE gel was gently agitated in a transfer buffer $(25 \mathrm{mM}$ Tris, $\mathrm{pH} 7.6,20 \%$ methanol, $40 \mathrm{mM}$ 6-amino- $n$-caproic acid, $0.02 \%$ SDS) containing $1 \mathrm{mM}$ EDTA for $10 \mathrm{~min}$, and subsequently resuspended in another buffer without EDTA for $10 \mathrm{~min}$ before electroblotting. After SDS-PAGE, proteins were electroblotted onto a polyvinylidene fluoride membrane (ATTO, Tokyo, Japan) according to the manufacturer's instructions. Specific antibodies against plant proteins were obtained from Agrisera (Vännas, Sweden). Phospho-Thr antibody was obtained from Cell signaling technology (Beverly, MA, USA). Alkaline phosphatase-conjugated anti-rabbit or anti-mouse IgG antibody (Sigma-Aldrich, St. Louis, MO, USA) was used as a secondary antibody, and signals were visualized by bromo-chloro-indolyl phosphate and nitroblue tetrazolium. Densitometric analysis was carried out using ImageJ software (version 1.41, NIH, Bethesda, MD, USA) after scanning the blotted membrane.

\subsection{Protein Identification by Mass Spectrometry}

The thylakoid membrane fractions from wheat plants, heat-stressed at $40{ }^{\circ} \mathrm{C}$ for $30 \mathrm{~min}$ in the presence of light, were prepared with $20 \mathrm{mM}$ Tricine- $\mathrm{NaOH}, \mathrm{pH} 7.9,0.4 \mathrm{M}$ sucrose, and $10 \mathrm{mM} \mathrm{NaCl}$, and then phosphorylated proteins were purified by immobilized metal affinity chromatography (IMAC) using a ProQ Phosphorylated Protein Purification Kit [40] (Invitrogen, Waltham, MA, USA). Phosphorylated protein fraction was separated by SDS-PAGE and stained with Coomassie Brilliant Blue. Next, two gel slices were excised from a sample lane in the $23-37 \mathrm{kDa}$ range, followed by in-gel digestion with $10 \mu \mathrm{g} / \mathrm{mL}$ trypsin (Promega, Fitchburg, WI, USA) overnight at $37{ }^{\circ} \mathrm{C}$ [41]. Digested 
peptides were eluted with $0.1 \%$ formic acid and were subjected to LC-MS/MS analysis, which was performed on an LCMS-IT-TOF (Shimadzu, Kyoto, Japan) interfaced with a nano reverse-phase liquid chromatography system (Shimadzu, Kyoto, Japan). LC separation was performed using a Pico Frit Beta Basic C18 column (New Objective, Woburn, MA, USA) at $300 \mathrm{~nL} / \mathrm{min}$. Peptides were eluted using gradients of $5 \%-45 \%$ acetonitrile in $0.1 \%$ formic acid and sprayed directly into the mass spectrometer. The heated capillary temperature and electrospray voltage were set at $200{ }^{\circ} \mathrm{C}$ and $2.5 \mathrm{kV}$, respectively. MS/MS data were acquired in the data-dependent mode by LCMS solution software (Shimadzu, Kyoto, Japan) and were converted to a single text file (containing the observed precursor peptide $\mathrm{m} / \mathrm{z}$, the fragment ion $\mathrm{m} / \mathrm{z}$, and intensity values) by Mascot Distiller (Matrixscience, Boston, MA, USA). The file was analyzed using the Mascot (Matrixscience) MS/MS Ion Search to search, and the obtained peptides were assigned to the SwissProt database (SIB, Geneva, Switzerland). The search parameters were set as follows: Database, SwissProt; Taxonomy, all; Enzyme, trypsin; Variable modifications, carbamidomethyl (C), oxidation (M), propionamid (C); Peptide tol., \pm 0.05 Da; and MS/MS tol., \pm 0.05 Da. For protein identification, the criteria were as follows: (1) Mascot scores above the statistically significant threshold $(p<0.05)$ and $(2)$ at least one top-ranked unique peptide matching the identified protein.

\subsection{Blue Native-PAGE}

Blue native-PAGE was performed as described previously in [35,42], with some minor modifications. Isolated thylakoid membranes were solubilized in $25 \mathrm{mM}$ BisTris- $\mathrm{HCl}(\mathrm{pH}$ 7.0), $20 \%$ glycerol, $0.25 \mathrm{mg} / \mathrm{mL}$ Pefabloc, $0.1 \% \alpha$ - $n$-dodecyl-D-maltoside and $1 \%$ digitonin at a final chlorophyll concentration of $0.5 \mathrm{mg} / \mathrm{mL}$. After incubation for $20 \mathrm{~min}$ on the ice and centrifugation at $18,000 \times g$ for another $20 \mathrm{~min}$, the supernatants were supplemented with $1 / 10$ volume of BN sample buffer (100 mM BisTris-HCl, pH 7.0, 5\% Serva blue G, 0.5 M 6-amino- $n$-caproic acid, and 30\% sucrose $(w / v))$, as established previously [43]. Thylakoid protein complexes were separated by NativePAGETM Novex ${ }^{\circledR} 3 \%-12 \%$ Bis-Tris Protein Gels (Invitrogen, Carlsbad, CA, USA).

\subsection{Transmission Electron Microscopy}

Leaves were trimmed into small pieces $\left(2 \times 3 \mathrm{~mm}^{2}\right)$ with a razor blade; these pieces were prefixed with $2.5 \%$ glutaraldehyde (Nisshin EM, Tokyo, Japan) in $0.1 \mathrm{M}$ phosphate-buffered saline (PBS) at $\mathrm{pH} 7.4$ at $4{ }^{\circ} \mathrm{C}$, overnight. Leaf pieces were then rinsed with PBS three times at intervals of 10 min and postfixed with $1 \%$ buffered osmium tetroxide (Nisshin EM, Tokyo, Japan) at room temperature for $1 \mathrm{~h}$. After the samples had been rinsed briefly with distilled water, they were immediately dehydrated in ethanol series $(50 \%, 70 \%, 90 \%$, and 100\%). They were then immersed in an intermediate solvent (propylene oxide; Nisshin EM, Tokyo, Japan) for $10 \mathrm{~min}$ and in a mixture $(1: 1, v / v)$ of propylene oxide and Spurr's resin [44] (Polysciences, Warrington, PA, USA) for $6 \mathrm{~h}$ at room temperature and then placed in pure Spurr's resin at $4{ }^{\circ} \mathrm{C}$ for 3 days, and then embedded in plain embedding plates and polymerized at $70{ }^{\circ} \mathrm{C}$ for $24 \mathrm{~h}$.

Blocks of leaves were cut with a Porter-Blum MT-1 ultramicrotome (Ivan Sorvall, Norwalk, CT, USA) and a diamond knife (Diatome, Bienne, Switzerland). Ultrathin sections about $80 \mathrm{~nm}$ were prepared; these were stained with 4\% aqueous UA for $10 \mathrm{~min}$ at room temperature and Sato's lead for 10 min [45]. 
Every staining step was succeeded by a step of washing with water three times for 3 min. The stained as well as the unstained sections were observed under a JEM-1400 electron microscope (JEOL, Akishima, Japan) at an accelerating voltage of $75 \mathrm{kV}$.

\section{Conclusions}

Photosystems of higher plants alleviate heat-induced damage in the presence of light under moderate stressed conditions. To obtain a better understanding of heat tolerance of the photosystem, we focused on the regulating photochemical energy transfer in heat-treated wheat at $40{ }^{\circ} \mathrm{C}$ with light. Structural changes of thylakoid membrane accompanied by the phosphorylation of thylakoid proteins such as D1 and D2 proteins and the LHCII proteins Lhcb1 and Lhcb2 would assist the remodeling of photosystems and regulation of energy distribution by transition toward state 2 probably contributes to plastoquione oxidation; thus, light-driven electrons flowing through PSI play a protective role against PSII damage under heat stress. In this research, state 1 to state 2 transition was observed under high temperature in light. Further research is expected to elucidate the detailed mechanism of heat stress-induced state transition e.g., distinguish between light intensity-induced and heat stress-induced state transition.

\section{Supplementary Materials}

Supplementary table can be found at http://www.mdpi.com/1422-0067/15/12/23042/s1.

\section{Acknowledgments}

We acknowledge the Integrated Center for Mass Spectrometry, Kobe University, Graduate School of Medicine for their assistance in determination of phosphorylated proteins. We thank Dr. N. Ohnishi for helpful discussions. This work was supported by Grant-in-Aids for Scientific Research (Yasuo Yamauchi, 23580456) from the Ministry of Education, Culture, Sports, Science and Technology, Japan, and JSPS Research Fellowship for Young Scientists (Yoko Marutani, 251843).

\section{Author Contributions}

Yoko Marutani and Yasuo Yamauchi conceived and designed the experiments; Yoko Marutani, Akihito Miyoshi and Kanako Inoue performed the experiments; Ken-ichi Ikeda, Masaharu Mizutani and Yukihiro Sugimoto analyzed the data; Yoko Marutani and Yasuo Yamauchi wrote the paper.

\section{Conflicts of Interest}

The authors declare no conflict of interest.

\section{References}

1. Sharkey, T.D.; Zhang, R. High Temperature Effects on Electron and Proton Circuits of Photosynthesis. J. Integr. Plant Biol. 2010, 52, 712-722. 
2. Eckardt, N.A.; Portis, A.R., Jr. Heat Denaturation Profiles of Ribulose-1,5-Bisphosphate Carboxylase/Oxygenase (Rubisco) and Rubisco Activase and the Inability of Rubisco Activase to Restore Activity of Heat-Denatured Rubisco. Plant Physiol. 1997, 113, 243-248.

3. Santarius, K.; Müller, M. Investigations on heat resistance of spinach leaves. Planta 1979, 146, 529-538.

4. Strasser, B. Donor side capacity of Photosystem II probed by chlorophyll a fluorescence transients. Photosynth. Res. 1997, 52, 147-155.

5. Cao, J.; Govindjee. Chlorophyll a fluorescence transient as an indicator of active and inactive Photosystem II in thylakoid membranes. Biochim. Biophys. Acta 1990, 1015, 180-188.

6. Havaux, M. Rapid photosynthetic adaptation to heat stress triggered in potato leaves by moderately elevated temperatures. Plant Cell Environ. 1993, 16, 461-467.

7. Heckathorn, S.A.; Downs, C.A.; Coleman, J.S. Small Heat Shock Proteins Protect Electron Transport in Chloroplasts and Mitochondria during Stress. Am. Zool. 1999, 39, 865-876.

8. Heckathorn, S.A.; Downs, C.A.; Sharkey, T.D.; Coleman, J.S. The Small, Methionine-Rich Chloroplast Heat-Shock Protein Protects Photosystem II Electron Transport during Heat Stress. Plant Physiol. 1998, 116, 439-444.

9. Peñuelas, J.; Llusià, J.; Asensio, D.; MunnÉ-Bosch, S. Linking isoprene with plant thermotolerance, antioxidants and monoterpene emissions. Plant Cell Environ. 2005, 28, 278-286.

10. Sharkey, T.D. Effects of moderate heat stress on photosynthesis: Importance of thylakoid reactions, rubisco deactivation, reactive oxygen species, and thermotolerance provided by isoprene. Plant Cell Environ. 2005, 28, 269-277.

11. Velikova, V.; Loreto, F. On the relationship between isoprene emission and thermotolerance in Phragmites australis leaves exposed to high temperatures and during the recovery from a heat stress. Plant Cell Environ. 2005, 28, 318-327.

12. Chaitanya, K.V.; Sundar, D.; Masilamani, S.; Ramachandra Reddy, A. Variation in heat stress-induced antioxidant enzyme activities among three mulberry cultivars. Plant Growth Regul. 2002, 36, 175-180.

13. Minagawa, J. State transitions-The molecular remodeling of photosynthetic supercomplexes that controls energy flow in the chloroplast. Biochim. Biophys. Acta Bioenerg. 2011, 1807, 897-905.

14. Wientjes, E.; van Amerongen, H.; Croce, R. LHCII is an antenna of both photosystems after long-term acclimation. Biochim. Biophys. Acta Bioenerg. 2013, 1827, 420-426.

15. Nellaepalli, S.; Mekala, N.R.; Zsiros, O.; Mohanty, P.; Subramanyam, R. Moderate heat stress induces state transitions in Arabidopsis thaliana. Biochim. Biophys. Acta Bioenerg. 2011, 1807, 1177-1184.

16. Ducruet, J.M.; Peeva, V.; Havaux, M. Chlorophyll thermofluorescence and thermoluminescence as complementary tools for the study of temperature stress in plants. Photosynth. Res. 2007, 93, 159-171.

17. Mohanty, P.; Vani, B.; Prakash, J.S. Elevated temperature treatment induced alteration in thylakoid membrane organization and energy distribution between the two photosystems in Pisum sativum. Z. Naturforsch. C 2002, 57, 836-842. 
18. Yamauchi, Y.; Kimura, Y. Photosystem at High Temperature-Mechanisms of Adaptation and Damage. In Photochemistry: UV/VIS Spectroscopy, Photochemical Reactions and Photosynthesis, 1st ed.; Maes, K.J., Willems, J.M., Eds.; NOVA Science: Hauppauge, NY, USA, 2011; pp. 203-236.

19. Marutani, Y.; Yamauchi, Y.; Kimura, Y.; Mizutani, M.; Sugimoto, Y. Damage to photosystem II due to heat stress without light-driven electron flow: Involvement of enhanced introduction of reducing power into thylakoid membranes. Planta 2012, 236, 753-761.

20. Havaux, M.; Tardy, F. Temperature-dependent adjustment of the thermal stability of photosystem II in vivo: Possible involvement of xanthophyll-cycle pigments. Planta 1996, 198, 324-333.

21. Bukhov, N.; Wiese, C.; Neimanis, S.; Heber, U. Heat sensitivity of chloroplasts and leaves: Leakage of protons from thylakoids and reversible activation of cyclic electron transport. Photosynth. Res. 1999, 59, 81-93.

22. Allen, J.F.; Forsberg, J. Molecular recognition in thylakoid structure and function. Trends Plant Sci. 2001, 6, 317-326.

23. Chuartzman, S.G.; Nevo, R.; Shimoni, E.; Charuvi, D.; Kiss, V.; Ohad, I.; Brumfeld, V.; Reich, Z. Thylakoid membrane remodeling during state transitions in Arabidopsis. Plant Cell 2008, 20, 1029-1039.

24. Dreier, W.; Schnarrenberger, C.; Börner, T. Light- and Stress-Dependent Enhancement of Amylolytic Activities in White and Green Barley Leaves: $\beta$-Amylases are Stress-Induced Proteins. J. Plant Physiol. 1995, 145, 342-348.

25. Zhang, R.; Wise, R.; Struck, K.; Sharkey, T. Moderate heat stress of Arabidopsis thaliana leaves causes chloroplast swelling and plastoglobule formation. Photosynth. Res. 2010, 105, 123-134.

26. Bellafiore, S.; Barneche, F.; Peltier, G.; Rochaix, J.-D. State transitions and light adaptation require chloroplast thylakoid protein kinase STN7. Nature 2005, 433, 892-895.

27. Fristedt, R.; Willig, A.; Granath, P.; Crevecoeur, M.; Rochaix, J.D.; Vener, A.V. Phosphorylation of photosystem II controls functional macroscopic folding of photosynthetic membranes in Arabidopsis. Plant Cell 2009, 21, 3950-3964.

28. Kinoshita, E.; Kinoshita-Kikuta, E.; Takiyama, K.; Koike, T. Phosphate-binding Tag, a New Tool to Visualize Phosphorylated Proteins. Mol. Cell. Proteomics 2006, 5, 749-757.

29. Leoni, C.; Pietrzykowska, M.; Kiss, A.Z.; Suorsa, M.; Ceci, L.R.; Aro, E.-M.; Jansson, S. Very rapid phosphorylation kinetics suggest a unique role for Lhcb2 during state transitions in Arabidopsis. Plant J. 2013, 76, 236-246.

30. Pietrzykowska, M.; Suorsa, M.; Semchonok, D.A.; Tikkanen, M.; Boekema, E.J.; Aro, E.-M.; Jansson, S. The Light-Harvesting Chlorophyll a/b Binding Proteins Lhcb1 and Lhcb2 Play Complementary Roles during State Transitions in Arabidopsis. Plant Cell 2014, 26, 3646-3660.

31. Shapiguzov, A.; Ingelsson, B.; Samol, I.; Andres, C.; Kessler, F.; Rochaix, J.D.; Vener, A.V.; Goldschmidt-Clermont, M. The PPH1 phosphatase is specifically involved in LHCII dephosphorylation and state transitions in Arabidopsis. Proc. Natl. Acad. Sci. USA 2010, 107, 4782-4787.

32. Pribil, M.; Pesaresi, P.; Hertle, A.; Barbato, R.; Leister, D. Role of plastid protein phosphatase TAP38 in LHCII dephosphorylation and thylakoid electron flow. PLoS Biol. 2010, 8, e1000288. 
33. Samol, I.; Shapiguzov, A.; Ingelsson, B.; Fucile, G.; Crevecoeur, M.; Vener, A.V.; Rochaix, J.D.; Goldschmidt-Clermont, M. Identification of a Photosystem II Phosphatase Involved in Light Acclimation in Arabidopsis. Plant Cell 2012, 24, 2596-2609.

34. Galka, P.; Santabarbara, S.; Khuong, T.T.; Degand, H.; Morsomme, P.; Jennings, R.C.; Boekema, E.J.; Caffarri, S. Functional analyses of the plant photosystem I-light-harvesting complex II supercomplex reveal that light-harvesting complex II loosely bound to photosystem II is a very efficient antenna for photosystem I in state II. Plant Cell 2012, 24, 2963-78.

35. Wientjes, E.; Drop, B.; Kouřil, R.; Boekema, E.J.; Croce, R. During State 1 to State 2 Transition in Arabidopsis thaliana, the Photosystem II Supercomplex Gets Phosphorylated but Does Not Disassemble. J. Biol. Chem. 2013, 288, 32821-32826.

36. Demmig-Adams, B.; Adams Iii, W.W.; Barker, D.H.; Logan, B.A.; Bowling, D.R.; Verhoeven, A.S. Using chlorophyll fluorescence to assess the fraction of absorbed light allocated to thermal dissipation of excess excitation. Physiol. Plant. 1996, 98, 253-264.

37. Quick, W.P.; Stitt, M. An examination of factors contributing to non-photochemical quenching of chlorophyll fluorescence in barley leaves. Biochim. Biophys. Acta Bioenerg. 1989, 977, 287-296.

38. Müller, P.; Li, X.-P.; Niyogi, K.K. Non-Photochemical Quenching. A Response to Excess Light Energy. Plant Physiol. 2001, 125, 1558-1566.

39. Khatoon, M.; Inagawa, K.; Pospíšil, P.; Yamashita, A.; Yoshioka, M.; Lundin, B.; Horie, J.; Morita, N.; Jajoo, A.; Yamamoto, Y.; et al. Quality Control of Photosystem II: Thylakoid unstacking is necessary to avoid further damage to the $\mathrm{d} 1$ protein and to facilitate $\mathrm{d} 1$ degradation under light stress in spinach thylakoids. J. Biol. Chem. 2009, 284, 25343-25352.

40. Schilling, M.; Knapp, D.R. Enrichment of phosphopeptides using biphasic immobilized metal affinity-reversed phase microcolumns. J. Proteome Res. 2008, 7, 4164-4172.

41. Hatano, N.; Hamada, T. Proteome Analysis of Pitcher Fluid of the Carnivorous Plant Nepenthes alata. J. Proteome Res. 2008, 7, 809-816.

42. Järvi, S.; Suorsa, M.; Paakkarinen, V.; Aro, E.M. Optimized native gel systems for separation of thylakoid protein complexes: Novel super- and mega-complexes. Biochem. J. 2011, 439, 207-214.

43. Peng, L.; Shimizu, H.; Shikanai, T. The Chloroplast NAD(P)H Dehydrogenase Complex Interacts with Photosystem I in Arabidopsis. J. Biol. Chem. 2008, 283, 34873-34879.

44. Spurr, A.R. A low-viscosity epoxy resin embedding medium for electron microscopy. J. Ultrastruct. Res. 1969, 26, 31-43.

45. Sato, T. A modified method for lead staining of thin sections. J. Electron Microsc. 1968, 17, $158-159$.

(C) 2014 by the authors; licensee MDPI, Basel, Switzerland. This article is an open access article distributed under the terms and conditions of the Creative Commons Attribution license (http://creativecommons.org/licenses/by/4.0/). 